\title{
LA CIENCIA DE LA CONCIENCIA SEGÚN STANISLAS DEHAENE
}

\author{
CONSCIOUSNESS SCIENCE ACCORDING TO STANISLAS DEHAENE
}

\author{
Alfredo Martínez Sánchez* \\ Grupo de investigación "Historia de la Filosofía en Andalucía", Universidad de Málaga, \\ Málaga - España.
}

Recibido septiembre de 2018/Received September, 2018

Aceptado noviembre de 2018/Accepted November, 2018

\begin{abstract}
RESUMEN
El objetivo de este artículo es doble. En primer lugar, contextualizar filosóficamente la investigación neurocientífica sobre la conciencia llevada a cabo por Stanislas Dehaene, tomando como referencia fundamental su obra Consciousness and the Brain. Deciphering how the brain codes our thoughts (2014), donde presenta un programa para una ciencia de la conciencia y una síntesis de una parte relevante de la investigación reciente sobre el tema. En relación con este objetivo se introducirá la distinción entre conciencia de acceso y conciencia fenomenológica, por una parte, y la distinción entre el problema fácil y el problema difícil de la conciencia, por otra. En segundo lugar, se discutirán críticamente algunas de las propuestas de Dehaene desde un punto de vista conceptual y epistemológico, en particular, las nociones de signatura de la conciencia y de decodificación o lectura cerebral.

Palabras Clave: Conciencia, Dehaene, correlatos neuronales de la conciencia, decodificación cerebral.
\end{abstract}

\begin{abstract}
The aim of this article is twofold. First, to philosophically contextualize the neuroscientific research on consciousness carried out by Stanislas Dehaene, taking as a fundamental reference his work Consciousness and the Brain. Deciphering how the brain codes our thoughts (2014), where he presents a program for a science of consciousness and a synthesis of a relevant part of recent research on the subject. In relation to this objective, the distinction between access consciussness and phenomenological consciousness will be introduced, on the one hand, and the distinction between the easy problem and the difficult problem of consciousness, on the other. Secondly, some of Dehaene's proposals will be critically discussed from a conceptual and epistemological point of view, in particular, the notions of signature of consciousness and brain decoding/mind reading.
\end{abstract}

Key Words: Consciousness, Dehaene, neural correlates of consciousness, brain decoding.

\section{Introducción}

Dehaene abre su libro Consciousness and the Brain. Deciphering how the brain codes our thoughts (2014) ${ }^{1}$ con el anuncio de que se propone examinar cómo un misterio filosófico se ha convertido en un fenómeno de laboratorio. De un modo menos retórico, y con más exactitud, presenta un programa para una ciencia de la conciencia y una síntesis de una parte relevante de la investigación reciente sobre la conciencia. En este sentido, la obra ofrece una ventana fascinante al estado actual de la ciencia de la conciencia desde una perspectiva determinada, destacando, entre otros aspectos, la explicación accesible, pero rigurosa, de diversos experimentos. Dehaene muestra a través de ellos la potencia explicativa de su teoría del espacio

* Autor correspondiente / Correspondig author: alms22@ hotmail.com 
de trabajo neuronal global (ETNG) -basada en la noción de espacio global de trabajo de B. Baars²-, con respecto a gran parte de las cuestiones que una ciencia de la conciencia debe afrontar, desde la identificación de los correlatos neuronales de la conciencia o la presencia de conciencia en animales y en máquinas hasta sus aplicaciones clínicas.

Debe quedar claro desde el principio que no existe una ciencia de la conciencia, sino distintos planteamientos teóricos y distintas líneas de investigación. Dehaene, sin embargo, logra armar una propuesta ambiciosa que incorpora tanto elementos empíricos como teóricos, de manera que unos y otros se iluminan mutuamente. Hay que recordar, además, que ni siquiera contamos con un concepto científico de conciencia. Por ese motivo, el primer ingrediente que para el autor debe tener una ciencia de la conciencia es el conceptual: la articulación de una mejor definición de conciencia. Puede considerarse que el trabajo conceptual sobre la conciencia proporciona un buen ejemplo de cómo filosofía y ciencia pueden colaborar, lo que reflejaría una orientación mucho más interesante que el sesgo positivista implícito en las declaraciones de numerosos neurocientíficos -y de algunos filósofos-, sugiriendo algo así como que la ciencia va a poner definitivamente fin a un presunto oscurantismo filosófico. Aunque es cierto que la conciencia ha entrado en el laboratorio, y eso es una buena noticia, la ciencia de la conciencia está lejos de proporcionar una explicación completa, en términos meramente científicos, y exhaustiva, en términos antropológicos ${ }^{3}$.

Como se pondrá de manifiesto más adelante, la determinación del concepto de conciencia como conciencia de acceso en Dehaene implica dos decisiones. Por una parte, una opción filosófica y empíricamente controvertida dentro de un debate aún abierto acerca de la distinción entre conciencia de acceso y conciencia fenoménica, y por otra, restringir la investigación a una dimensión básica y limitada de la conciencia: la percepción.

El autor presenta, además del conceptual, otros dos factores que hacen posible una ciencia de la conciencia, a saber, el descubrimiento de que la conciencia puede ser manipulada experimentalmente, y un nuevo respeto por los fenómenos subjetivos. A estos tres factores hay que añadir un cuarto elemento crucial en la propuesta de Dehaene: las signaturas o marcadores de la conciencia, entendidos como patrones de actividad cerebral que aparecen únicamente cuando el sujeto tiene una experiencia consciente ${ }^{4}$. Este hallazgo empírico es completado y explicado mediante la propuesta teórica ya mencionada: la teoría del ETNG, según la cual la conciencia es información global difundida en el cortex gracias a la interconexión de distintas redes neuronales.

Como veremos, y ya se ha adelantado con respecto al concepto de conciencia, ninguno de estos cuatro elementos está exento de controversia. En la sección I me ocuparé del primer elemento, el concepto de conciencia, procurando contextualizar filosóficamente la noción de conciencia de acceso dentro del debate acceso/fenomenología (conectado con la cuestión de si existe un problema difícil de la conciencia) ${ }^{5}$. En la sección II consideraré las signaturas de la conciencia, lo que dará pie a una revisión de algunas de las ideas esenciales del autor.

\section{El Concepto de Conciencia. Conciencia de Acceso y Conciencia Fenoménica}

En la ciencia contemporánea de la conciencia se distinguen, según Dehaene, al menos tres conceptos de conciencia: estado de vigilia, atención, y conciencia de acceso $^{6}$. La conciencia de acceso tiene su modelo en la percepción: la información que llega a nuestros sentidos es inicialmente procesada de manera inconsciente, pero cuando esta información es difundida globalmente en el cerebro de manera que puede ser utilizada para diversos propósitos, como la acción o el informe verbal, se pasa al procesamiento consciente y se produce la correspondiente experiencia subjetiva, el sujeto es entonces capaz de informar, de algún modo, sobre si percibe un determinado estímulo. Este acceso consciente constituye el tipo de conciencia del que Dehaene primordialmente se ocupa $\mathrm{y}$ al que considera como genuino. A lo largo del libro hablar de conciencia equivale normalmente a hablar de acceso consciente, aunque en el capítulo 6, al tratar del estado vegetativo, del coma, y de otras situaciones relacionados, se considera la conciencia en el primer sentido.

La conciencia de acceso sería, a su vez, una vía para formas más complejas de conciencia, por ejemplo, el sentido de sí o de sí-mismo. El modo en que el autor plantea la relación entre conciencia y sí-mismo puede suscitar alguna confusión. Por ejemplo, puede llevar a malinterpretar la concepción de Damasio, que aparece explícitamente citado (p. 23), si se entiende su noción 
de sí-mismo (self) desde el punto de vista de la conciencia de acceso, es decir, como un contenido al que se accede. Es cierto que puede haber una forma metacognitiva y reflexiva del sí-mismo, una forma de autoconciencia o autoconocimiento, pero el sí-mismo implicado necesariamente en la conciencia del que habla Damasio no se refiere al sí-mismo como un contenido de conciencia al que se accede, comparable a una percepción (una especie de autorrepresentación), sino que la propia percepción en tanto que experiencia subjetiva se experimenta de ese modo, es decir, es la forma en que la experiencia subjetiva se da. Desde este punto de vista, solo en el caso del sí-mismo metacognitivo como reflexión o autoconocimiento se podría taxativamente asegurar, como hace Dehaene, que la conexión entre percepción consciente y sí-mismo es innecesaria ${ }^{7}$.

Para contextualizar filosóficamente la noción de conciencia de acceso debemos considerar el concepto de conciencia fenoménica, al que, sin embargo, el autor solo alude brevemente. La conciencia fenoménica es frecuentemente entendida como experiencia subjetiva (la experiencia de lo que algunos han llamado qualia, que, en un sentido amplio, remite a cómo nos aparece el mundo, desde la agudeza de un dolor de muelas hasta el verdor de la hierba). Dehaene señala que para algunos filósofos la experiencia subjetiva de los qualia no puede ser reducida a una descripción científica neuronal. Sin embargo, para el autor, la noción de conciencia fenoménica es una noción profundamente engañosa que conduce a una deriva dualista. Dehaene sostiene que mediante la clarificación de cómo la información sensorial accede a nuestra mente y se hace comunicable, es decir, mediante el estudio de la conciencia de acceso, el problema de la conciencia fenoménica simplemente se disolverá (p. 10). De hecho, afirma que David Chalmers (1999) ha invertido los términos: lo que Chalmers llama el problema difícil sería en realidad el problema fácil, y el problema fácil es realmente el difícil. En términos de Chalmers, el problema fácil incluye problemas técnicos para la ciencia cognitiva y algunos problemas conceptuales pero no plantea ningún "profundo enigma metafísico" (Chalmers, 1999, p. 49). El aprendizaje y la memoria, por ejemplo, serían, en este sentido, problemas fáciles. No porque el estudio científico de los mismos carezca de dificultades, sino porque los aspectos fenoménicos de la mente representan un reto diferente vinculado al problema mentecuerpo. Este reto no solo implica la cuestión de cómo un sistema físico puede originar la experiencia consciente, sino, específicamente, explicar por qué y cómo las propiedades psicológicas van acompañadas de propiedades fenoménicas.

Mi intención en este artículo no es tratar de resolver una polémica de la magnitud del debate sobre conciencia de acceso y conciencia fenoménica (problema fácil/difícil, función cognitiva/experiencia consciente $)^{8}$, sino contextualizar filosóficamente la noción de conciencia de acceso, poner de manifiesto algunas de las cuestiones en juego más relevantes, y mostrar, por una parte, que la propuesta de Dehaene está más abierta a la controversia de lo que él sugiere y, por otra, que el avance empírico no ha acabado, en contra de lo afirmado por el autor, con el debate filosóficamente pertinente.

Según Dehaene, una vez que el problema fácil se haya entendido se desvanecerá el supuestamente difícil (p. 262). Lo que el autor afirma no es que no exista cierta dimensión fenoménica de la experiencia subjetiva, sino que puede ser explicada científicamente del mismo modo que otros aspectos de la experiencia, asumiendo la posición de que esa dimensión fenoménica desempeña un papel cognitivo-funcional. Pero puede que en el planteamiento de Dehaene se encuentre cierta equivocidad. Una cuestión es si la dimensión cualitativa o fenoménica de la experiencia, lo que algunos han llamado qualia, puede ser explicada científicamente, otra cuestión es si puede ser explicada ahora, es decir, en el estado actual de la ciencia de la conciencia, y una tercera cuestión es si la explicación del acceso es suficiente para comprender la dimensión cualitativo/fenoménica o para hacer esta explicación innecesaria. Si se acepta que existe una dimensión cualitativo/fenoménica de la experiencia se debería aceptar que una ciencia de la conciencia debe proporcionar una explicación de esa dimensión. Parafraseando a Fahrenfort y Lamme (2012), una auténtica ciencia de la conciencia debería explicar la fenomenología. El problema es determinar, a partir de ahí, y dicho de manera sintética, si la explicación del acceso es suficiente para comprender la fenomenología. Fahrenfort y Lamme (2012), por ejemplo, consideran que el acceso por sí mismo no parece estar involucrado en la generación del contenido de la 
experiencia, y que, por tanto, tiene poco poder explicativo con respecto a la fenomenología.

La distinción entre conciencia de acceso y conciencia fenoménica viene siendo objeto de un largo debate que enfrenta a filósofos como Ned Block y neurocientíficos como Victor Lamme ${ }^{9}$ a autores como Dehaene y Daniel Dennett. Inicialmente, la distinción de Block fue fundamentalmente conceptual, aunque posteriormente se ha inclinado por la discusión sobre argumentos empíricos. Con objeto de aclarar el concepto de conciencia Block proponía distinguir entre conciencia fenoménica (lo que más arriba hemos caracterizado como qualia en un sentido amplio) y conciencia de acceso, que se definía por la accesibilidad para su uso en el razonamiento y para la guía racional del habla (reportabilidad, en términos generales) y de la acción (Block, 1995). Block entendía las propiedades de la conciencia fenoménica como algo completamente distinto de cualquier propiedad cognitiva, intencional o funcional. Coincidiendo con el problema difícil de Chalmers, es la conciencia fenoménica la que representaría un misterio para la ciencia, no la conciencia de acceso. Es la conciencia fenoménica la que marcaría un límite, o al menos una dificultad adicional, para la explicación científica de la conciencia, suscitando, así, una "brecha explicativa" (Levine, 1983). Buena parte de los argumentos de Block se han dirigido a mostrar casos de conciencia fenoménica sin conciencia de acceso y a justificar el llamado "argumento del desborde", según el cual la conciencia fenoménica desborda o excede la conciencia de acceso, en otras palabras, que la conciencia perceptiva es más rica que la conciencia de acceso (Block, 2011). Para Dehaene, sin embargo, no hay experiencia consciente más allá, o fuera, de la conciencia de acceso.

Un problema fundamental en este debate, que quizás no pueda ser zanjado empíricamente, consiste en decidir si tiene sentido hablar de experiencia a la que el sujeto no tiene acceso, y si lo que permanece como información disponible (accesible), pero sin llegar a entrar en el ámbito del acceso, debe ser considerado como experiencia consciente o como procesamiento inconsciente. Block (2012) entiende que la información accesible pero no accedida es consciente (menos clara es su posición con respecto a información "inaccesible"), mientras que Dehaene la incluye dentro de su tipología de lo inconsciente, concretamente, en la categoría que denomina preconsciente (p. 191). Por el momento, no parece haber evidencia empírica que incline claramente la balanza y el debate permanece abierto, pero la idea de una conciencia desconocida para el sujeto (una experiencia consciente que el sujeto niega tener, es decir, sin acceso) resulta manifiestamente anti-intuitiva.

Según Dehaene, el concepto de qualia entendido como pura experiencia mental completamente independiente del procesamiento de la información es una idea precientífica (la compara con el vitalismo), y el avance de las explicaciones en términos de lo que Chalmers llama el problema fácil acabará por explicar la experiencia subjetiva ${ }^{10}$. Si bien Dehaene reconoce la enorme capacidad del cerebro para realizar complejas operaciones de manera inconsciente y señala, dentro de la tendencia generalizada en las ciencias cognitivas, que normalmente sobrevaloramos el poder de la conciencia, encuentra en ella cruciales funciones cognitivas. La conciencia no es un mero epifenómeno, sino una propiedad biológica que emergió evolutivamente porque era útiil ${ }^{11}$. El término función debe entenderse en este contexto en este sentido, como respuesta a la pregunta "¿para qué sirve la conciencia?", enfocada desde un punto de vista evolutivo y psicológico -dándole un papel dentro de lo que Chalmers llama el problema fácil-, y no debe confundirse con su uso en filosofía de la mente en relación con la cuestión de la naturaleza e individuación de los estados mentales y de su contenido. La conciencia ocupa un nicho cognitivo específico y afronta problemas que el sistema de la mente inconsciente no puede tratar adecuadamente. Mientras que la información subliminal puede ser evanescente y fugaz, la consciente puede ser más estable, la conciencia condensa y selecciona la enorme cantidad de información que llega al cerebro y el resultado de esta selección es conducido hacia otras fases de procesamiento ${ }^{12}$. Esta función de difusión de la información es esencial, y en humanos es aumentada mediante el lenguaje que, a su vez, permite su prolongación en el plano social y cultural.

Sin duda, la explicación de para qué sirve la conciencia, de su función en este sentido, es una de las cuestiones que debe afrontar una ciencia de la conciencia y la propuesta de Dehaene merece ser tomada en consideración. El autor no niega que la dimensión cualitativo/fenomenológica 
deba ser explicada, lo que niega es la existencia de un problema especial o difícil con respecto a esa dimensión, la existencia de algún tipo de brecha explicativa y, por tanto, defiende lo que podríamos llamar una continuidad explicativa. Es en este sentido, sostengo, que hay que interpretar afirmaciones como "la ciencia de la conciencia irá absorbiendo y reduciendo el problema difícil hasta que desaparezca" y "[1]a ciencia de la conciencia ya explica significativas porciones de nuestra experiencia subjetiva, y no veo límites claros para este enfoque" (p. 262). Las preguntas pertinentes aquí serían: ¿Debe una ciencia de la conciencia explicar la fenomenología, la dimensión cualitativasubjetiva de la experiencia? ¿Ofrece la propuesta de Dehaene esa explicación? Si la respuesta a la primera pregunta es afirmativa, como creo que debe ser, entonces la propuesta del autor resulta, en este sentido, incompleta.

La noción de conciencia de acceso tiene la virtud de ser experimentalmente útil y científicamente abordable mediante el estudio de las condiciones en que un estímulo es, bien efectivamente percibido, bien simplemente procesado de manera inconsciente. Sin embargo, a pesar de la confianza que muestra el autor, no hay nada en su examen del acceso consciente, ni en el de los estados de la conciencia (vigilia, estado vegetativo, etc.), que proporcione una indicación significativa de cómo el estudio de los mismos y del tratamiento de la información en el espacio global, puede conducir a la explicación de la fenomenología y, en este sentido, a la disolución de lo que algunos llaman el problema difícil de la conciencia. Paralelamente, tampoco parece haber zanjado el debate entre teorías disociativas (que distinguen experiencia y acceso -Cohen y Dennett, 2011-) y teorías (solo) del acceso (ver nota 8).

\section{Las Signaturas De La Conciencia}

El contraste experimental entre la percepción consciente y la inconsciente (el segundo factor de una ciencia de la conciencia) resulta crucial en la propuesta de Dehaene, pues es a través de este contraste que se abre, según el autor, la posibilidad de identificar los mecanismos cerebrales distintivos de la percepción consciente. Dichos mecanismos, que el autor denomina signaturas o marcas de la conciencia, serían los patrones de actividad neuronal asociados exclusivamente con la experiencia consciente; una especie de criterios cerebrales de la conciencia. Encontrar esta signatura resulta así una tarea esencial en el proyecto de Dehaene, y la mayor parte del libro está dedicada a desarrollar y justificar tales criterios o marcadores junto con la teoría en la que se encuadran; la teoría del ETNG. Al estudiar la dimensión neurobiológica del espacio global de trabajo Dehaene convierte en neurocognitiva la que, en principio, era solo una teoría cognitiva. Esta concepción implica, como ya he mencionado, una visión funcional de la conciencia, en el sentido de que desempeña un papel preciso en la economía computacional del cerebro; en concreto, el de seleccionar, amplificar y propagar determinados contenidos ${ }^{13}$.

$\mathrm{El}$ autor distingue cuatro marcadores de la conciencia que, muy sintéticamente, serían:

1) Una amplificación de la actividad cerebral sensorial que resulta en una súbita "ignición" de circuitos parietales y frontales.

2) La aparición de una onda de tipo P3, registrada mediante encefalograma, un tercio de segundo después de que el estímulo haya sido presentado.

3) Una explosión tardía y súbita (no su mera presencia) de oscilaciones de alta frecuencia.

4) Una sincronización de intercambios de información entre regiones distantes del cerebro.

Como ilustración describiré brevemente la primera signatura. Mientras que un estímulo subliminal activa solo circuitos especializados, el mismo estímulo conscientemente percibido produce una amplificación de la activación en las áreas sensoriales que se va fortaleciendo progresivamente hasta invadir distintas regiones de los lóbulos frontal y parietal. El término "ignición" designa esta amplificación y expansión de la actividad cerebral que se produce a partir de cierto umbral. Dehaene asocia esta propagación de la actividad neuronal con la distribución de la información que propugna la teoría del espacio global de trabajo.

Dehaene señala como referencia significativa del despegue de la ciencia de la conciencia los trabajos de F. Crick y Ch. Koch al comienzo de los años 90. Él mismo se sitúa en esta estela (p. 19), presentando sus tres factores fundamentales para una ciencia de la conciencia como una continuación del programa de Crick y Koch. Sin embargo, este programa implica cierta ambigüedad, reflejada en la noción de correlato neuronal de la conciencia (CNC). Crick y Koch (1990) utilizan esta expresión (CNC) en un sentido próximo al de 
la expresión signatura de la conciencia, tal y como es empleado por Dehaene, es decir, en el contexto del contraste mínimo entre lo que es percibido conscientemente y lo que no. En este sentido, el $\mathrm{CNC}$ es una característica general compartida por distintos contenidos y por diferentes modalidades perceptivas que tienen en común, precisamente, el hecho de entrar en la conciencia en vez de ser procesados solo de manera inconsciente. Pero, más frecuentemente, la noción de $\mathrm{CNC}$ remite a los correlatos de contenidos específicos de la conciencia (Chalmers, 2000; Crick \& Koch, 2003; Tononi \& Koch, 2008), es decir, correlatos de perceptos particulares. La diferencia entre estos dos modos de entender el significado del CNC debe quedar clara. Desde este punto de vista, no sería lo mismo inferir a partir de un proceso o de una determinada activación neuronal que el sujeto es consciente o está percibiendo un estímulo de manera consciente, que decodificar el contenido específico de esa percepción (por ejemplo, si está percibiendo una ventana o bien una puerta, o incluso en qué está pensando). Esta ambigüedad se reproduce en el subtítulo del libro de Dehaene Deciphering How the Brain Codes Our Thoughts ${ }^{14}$. Una manera útil de enfocar este problema consiste en distinguir entre correlatos independientes del contenido (correlatos inespecíficos: CI) y correlatos neuronales de la conciencia referidos o asociados al contenido concreto (correlatos específicos: $\mathrm{CE}$ ).

\section{Signaturas, Correlatos y "Lectura Cerebral"}

Toda la argumentación de Dehaene al presentar sus signaturas de la conciencia gira en torno a correlatos de la conciencia de acceso, básicamente, mediante la diferencia entre percibir un objeto -siendo capaz de informar de ello- y el mero procesamiento no consciente del estímulo. No obstante, en una de las secciones del capítulo 4 (Decoding a Conscious Thought) nos dice que la signatura de la conciencia codifica la experiencia subjetiva completa ${ }^{15}$, con todos los detalles de su contenido, sugiriendo que lo que está en discusión son los contenidos concretos de la experiencia subjetiva. Mientras que en el primer caso se trata de correlatos inespecíficos (CI), en el segundo estaríamos ante correlatos de tipo específico $(\mathrm{CE})^{16}$.

$\mathrm{Al}$ señalar la diferencia entre un mero correlato y una signatura de la conciencia, el autor advierte que lo que está buscando no es cualquier relación estadística entre la actividad cerebral y la percepción consciente (meras correlaciones -ver más arriba la nota 3-), sino un fenómeno cerebral que esté presente siempre que se dé la percepción consciente y ausente cuando no se dé tal percepción ${ }^{17}$, y añade -esto es lo que quiero subrayar aquí-: "y que codifica la experiencia subjetiva completa que la persona refiere" (p. 142). El problema se advierte, entre otros momentos, cuando ofrece un ejemplo de lo que es un "mero correlato", la liberación de norepinefrina, entendido como una señal inespecífica relacionada con el nivel de vigilancia, pero "desprovista de las distinciones detalladas que forman el tejido de nuestra vida mental" (p. 143) (este tejido es el contenido de la experiencia subjetiva, es decir, su contenido fenoménico). La liberación de norepinefrina sería, por tanto, un correlato inespecífico independiente del contenido concreto. Desde este punto de vista, podemos distinguir entre correlatos que serían condición necesaria pero no suficiente para estar consciente (nivel de conciencia: vigilancia, estar despierto, etc.), y correlatos como condición necesaria y suficiente para estar consciente. Estos segundos correlatos (necesarios y suficientes para un adecuado nivel de consciencia) serían correlatos necesarios para la fenomenología (qualia, experiencia), pero no tienen por qué ser suficientes.

En el discurso de Dehaene entran en juego, fundamentalmente, tres conceptos de conciencia: en primer lugar, conciencia de acceso (el hecho de ser consciente de algo), en segundo lugar, contenido de un estado de conciencia, y, finalmente, estados o niveles de conciencia (sueño, coma, estado vegetativo $)^{18}$. Una cosa es la cuestión de cómo se llega a tener una fenomenología (en general) y de si se tiene o no experiencia consciente (primer concepto), y otra cosa es el contenido de la misma, es decir, la cuestión de cómo se genera y determina el contenido concreto (segundo concepto) ${ }^{19}$. La primera cuestión es básicamente la cuestión del acceso, y la segunda es la que está relacionada con el proyecto de la lectura o decodificación cerebral al que el autor se refiere en este contexto (Haynes, 2009). Lo que quiero señalar es que la cuestión de las signaturas y la cuestión de la decodificación no son claramente o suficientemente distinguidas en la presentación de Dehaene.

Del mismo modo, podemos apreciar la existencia de tres tipos diferentes de CNC, dos de ellos genéricos $(\mathrm{CI})$, comunes en principio a todo 
ser consciente, y un tercero específico o individual. En primer lugar, tendríamos correlatos genéricos como condición necesaria de la conciencia, del tipo de la liberación de norepinefrina, en segundo lugar, correlatos también genéricos pero conectados con el hecho de tener experiencia subjetiva o fenomenología (aquí entrarían las cuatro signaturas mencionas anteriormente), y, finalmente, los correlatos vinculados a contenidos específicos (CE). Por ejemplo, como señalan De Graaf, Hsieh, y Sack (2012) (mencionando explícitamente a Dehaene entre otros autores que han encontrado una correlación entre la activación del cortex frontoparietal y la conciencia), si el cortex frontoparietal está activo en los procesos conscientes con independencia del contenido específico de la experiencia subjetiva concreta (es decir, aparece como un correlato invariable a través de distintos perceptos), es poco probable que tales áreas sean, sin más, el sustrato neural de tal experiencia, sino, más bien, un correlato inespecífico ${ }^{20}$.

Mientras que los correlatos inespecíficos (CI) solamente indicarían si hay conciencia (por ejemplo, si el estímulo es visible, conscientemente percibido), los correlatos específicos (CE) se corresponderían con contenidos específicos. El CI sería común a distintos contenidos, nos indicaría que tales contenidos son conscientes, pero no de qué contenidos se trata. Desde un punto de vista empírico, cabría determinar si un CI es completamente invariante, permaneciendo el mismo a través de distintos contenidos, o si hay variaciones o modulaciones, en cuyo caso habría que comprobar si se corresponden, y cómo se corresponden, con las variaciones en contenido ${ }^{21}$. Por otra parte, la especificidad del contenido, y las variaciones del mismo, pueden entenderse a diferentes niveles, con diferentes grados de precisión: desde rasgos generales, como la diferencia entre color y brillo, hasta diferencias concretas entre, por ejemplo, las caras de distintos individuos, pasando por la diferencia entre categorías de objetos, por ejemplo, entre la categoría "caras" y la categoría "casas"22.

La inclusión del tema de la decodificación de los contenidos de la conciencia dentro del capítulo dedicado a las signaturas de la conciencia (capítulo 4) y el correspondiente tratamiento del tema puede generar, por tanto, cierta confusión: por una parte, las signaturas de la conciencia se identifican mediante metodologías de contraste mínimo en términos de correlatos inespecíficos, sin embargo, la decodificación del contenido de la conciencia se refiere a correlatos específicos, y la metodología de preferencia en el campo de la decodificación como lectura cerebral no se basa en métodos contrastivos sino correlacionales y predictivos como explico más abajo (concretamente, mediante el uso de algoritmos y programas informáticos en el ámbito del aprendizaje automático o machine learning [Meyers, 2013]).

Veamos a continuación otro aspecto potencialmente confuso del uso de la noción de decodificación. Según el autor, el código consciente "contiene un registro completo de la experiencia del sujeto, con exactamente el mismo nivel de detalle que el de lo que la persona percibe" (p. 143). De modo que "si pudiéramos leer este código tendríamos acceso completo a todo el mundo interno de la persona" (p. 145). Nos encontramos aquí con el proyecto de lectura cerebral (a veces llamado también lectura mental) que tanto impacto popular viene cosechando. Según Dehaene, un estado subjetivo de la mente podría ser decodificado a partir de un estado del cerebro (p. 147). El autor apela a estudios sobre neuronas individuales que han hallado que ciertas neuronas reaccionan a fotografías, lugares, o personas específicas. Por ejemplo, se ha encontrado que una neurona reacciona específicamente a una fotografía de Bill Clinton, pero no a la imagen de otra persona (Quian Quiroga, Reddy, Kreiman, Koch, \& Fried, 2005).

En términos generales, en la noción de decodificación podemos distinguir dos sentidos diferentes, uno débil o analógico y otro fuerte o genuino. El concepto débil es fundamentalmente correlacional, mientras que el significado genuino implica la comprensión e interpretación del código. Comprender un código supone conocer sus reglas. No es lo mismo establecer la correlación de un signo con su significado y a partir de un determinado número de correlaciones intentar predecir otras correlaciones, es decir, inferir en base a anteriores correlaciones qué significado corresponderá a un determinado signo dado, que hallar el significado mediante la aplicación de las reglas de codificación-descodificación (lo que, en términos generales, equivaldría a conocer la

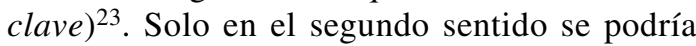
afirmar que se es capaz de entender el código y de, propiamente, leerlo. El primer procedimiento es solo aproximado y presenta limitaciones significativas para una lectura mental completa a partir 
de datos neurales. Por ejemplo, puede ocurrir que las correlaciones establecidas para un individuo tengan una capacidad predictiva relevante solo para ese individuo, o que dentro de un mismo individuo se formen nuevas correlaciones difíciles de predecir, debidas a nuevas experiencias y nuevos aprendizajes, al mismo tiempo que se deshacen asociaciones (correlaciones) antiguas. De hecho, el propio Dehaene señala que el código es único para cada persona y que puede variar en distintos momentos para un mismo individuo.

Uno de los autores que explícitamente han considerado el significado del concepto de decodificación ha sido J. D. Haynes (al que el propio Dehaene cita). En su propuesta, la decodificación viene a ser una especie de correlación cualificada, frente a otros tipos más débiles o genéricos de correlación: "El término decodificación (tal y como es usado aquí) se refiere a la mera capacidad de las técnicas estadísticas para comprobar si se da un mapeo sistemático entre estados de un CNN nuclear y una categoría de la experiencia" (Haynes, 2009 , p. 5$)^{24}$.

La razón para proponer el uso del término "codificación" en lugar de "correlación" no es para ir realmente más allá de la correlación sino simplemente para refinar y precisar el tipo de correlación relevante. En palabras de Heynes (2009), la razón es que la codificación proporciona un cuadro más general y más potente para identificar los correlatos neuronales nucleares de la conciencia (p. 2) ${ }^{25}$. Sin embargo, según el propio Haynes, la correlación perfecta implicada en las nociones de codificación y decodificación no es sino un ideal empírico debido a las limitaciones técnicas actuales. Además de estas limitaciones empíricas contingentes el autor examina una serie de puntos que ponen en cuestión una identificación simple y directa entre codificación y conciencia. En particular, señala ciertas dificultades para una teoría como la de Dehaene (ETNG). Esta teoría postula que la información codificada en regiones cerebrales de contenido y modalidad específicos no es suficiente y que la información debe ser distribuida globalmente a través del cerebro para alcanzar la conciencia, lo que supone la implicación de determinadas áreas prefrontales. Haynes indica la posibilidad de que la actividad en estas áreas refleje meramente procesos inespecíficos sin codificación de contenido específico.
La noción de signatura es presentada por Dehaene de un modo análogo a cómo Haynes introduce la noción de codificación, distinguiéndola de una noción débil de mera correlación estadística: la signatura se refiere a una actividad cerebral que está siempre presente cuando se produce percepción consciente y ausente cuando no se da tal percepción (además de tener un valor explicativo dentro de la teoría del ETNG). Pero una signatura es más un marcador que un correlato directo en sentido estricto, es decir, si un determinado fenómeno cerebral acompaña siempre el acceso consciente esto nos permitiría inferir la existencia de conciencia de acceso a partir de la presencia de tal fenómeno (marcador o signatura), pero eso no quiere decir que ese fenómeno constituya el sustrato o la base cerebral de la conciencia de acceso, y, menos aún, de la experiencia subjetiva específica ${ }^{26}$. Por ejemplo, de Graaf et al. (2012) (ver más arriba, nota 17) distinguen entre el sustrato que directamente soporta la experiencia consciente y otros correlatos que pueden ser un requisito, o bien una consecuencia. Aru, Bachmann, Singer y Melloni $(2012,2015)$ proponen la misma distinción y vinculan explícitamente este problema con el uso de la metodología contrastiva en la que se basa el trabajo de Dehaene ${ }^{27}$.

En este contexto, el hecho de que haya diversas signaturas de la conciencia ${ }^{28}$ puede significar dos cosas: o bien que hay diversos correlatos neuronales de la conciencia de acceso, o bien que las signaturas no son genuinos correlatos sino solamente un grupo de indicadores asociados a un único correlato. Además, si las signaturas deben ser entendidas como condiciones necesarias y suficientes, queda la pregunta de si todas son necesarias y suficientes. La posición de Dehaene es compleja. Por una parte, el correlato neuronal de la conciencia parece, a veces, concebido más en términos funcionales que estructurales o anatómi$\cos$, por lo que existe cierto grado de variabilidad en cuanto a la realización de la conciencia en una red neuronal distribuida, en la que, al parecer, no necesariamente están siempre implicados los mismos componentes -si bien parece que el cortex prefrontal es una región habitualmente incluida ${ }^{29}$-; por otra parte, las signaturas serían distintas manifestaciones de un solo proceso, vinculadas a la técnica de detección utilizada en cada caso (resonancia magnética en el caso de la ignición masiva de circuitos parietales y frontales, electroencefalografía en el 
caso de la onda P3, etc.). En la medida en que la conciencia consiste en una distribución global de la información, su sustrato neuronal implica la conexión entre distintas áreas del cerebro, pero de la propuesta de Dehaene es difícil inferir si hay una red neuronal mínima suficiente y necesaria, o cuál es la red mínimamente suficiente. Lo que sí parece que se puede concluir es que no existe una única región cerebral específica que constituya el sustrato material de la conciencia.

El trabajo de Quian Quiroga y colaboradores $(2005,2009)$ pone también de manifiesto el significado limitado (y hasta engañoso si se interpreta superficialmente) del paradigma de la decodificación. La predicción o inferencia de las imágenes que el sujeto ve depende de dos factores clave. Por una parte, se trata de imágenes de elementos familiares para el sujeto, por otra, no hay garantía de que la asociación neurona-objeto se mantenga de manera estable, como ya he comentado. Nadie nace con una neurona "para" Jenifer Aniston, obviamente, sino que esa asociación se debe a la experiencia individual. Por tanto, la misma neurona, y quizás el mismo patrón de activación, puede estar asociada a distintos objetos en distintas personas. No es, por tanto, posible observar directamente ( $\sin$ un estudio previo) el cerebro de cualquier persona que está viendo una imagen e inferir con seguridad y precisión qué imagen está viendo (eso sí sería genuina decodificación). Por otra parte, aún en el caso de un sujeto previamente estudiado con vistas a establecer las correlaciones relevantes para desarrollar los instrumentos de predicción correspondientes (algoritmos), es muy posible que el nivel de corrección de las predicciones disminuya con el tiempo, ya que, como hemos visto, nuevas experiencias pueden alterar las asociaciones forjadas a través de anteriores experiencias.

Dehaene trata de aplicar las signaturas de la conciencia en tres contextos diferentes. El primer contexto es el de la metodología contrastiva. Este estudio de la conciencia en términos de encendido/ apagado, por así decirlo, es el que da lugar a la propia identificación de tales signaturas. El segundo contexto es al que alude el subtítulo del libro, la decodificación del pensamiento o de la experiencia consciente, que aparece en el capítulo dedicado expresamente a las signaturas de la conciencia.
El tercer contexto es el que concierne a pacientes neurológicos, a bebés, y a animales no humanos. Por razones de espacio no me ocupo aquí de esta temática, pero una pregunta inevitable es si las signaturas de la conciencia establecidas en sujetos humanos adultos y normales permanecen tal cuales y con el mismo significado (o cómo deben de interpretarse las diferencias) cuando se trata de pacientes con alteraciones neuronales, animales no humanos y niños de menos de un año. En el planteamiento de Dehaene las signaturas tienden a ser entendidas como un criterio transversal, es decir, como un rasgo significativamente estable que permanece a través de importantes diferencias en lo que suele llamarse niveles, estados, o modos de conciencia. Estas diferencias también suscitan la cuestión de si los distintos estados de conciencia implican también diferencias en los contenidos de la conciencia y, si se da el caso, cómo se ve afectado el contenido de la conciencia ${ }^{30}$.

\section{Reflexiones Finales}

Para terminar, señalar que, además de las cuestiones ya mencionadas, las apuestas de Dehaene abren explícitamente otros frentes filosóficos controvertidos, como son una concepción internalista de la mente, la existencia de un lenguaje del pensamiento, una interpretación reduccionista de lo mental, la naturaleza de la libertad y su correspondiente concepción antropológica.

La idea de que ha llegado el momento en el que la ciencia nos va a librar definitivamente de un presunto oscurantismo filosófico puede ser considerada como una simplificación retórica o ideológica (o ambas). Aunque es cierto que la conciencia ha entrado en el laboratorio, y eso es una buena noticia, la ciencia de la conciencia está lejos de proporcionar una explicación completa y satisfactoria, tanto en términos meramente científicos como en términos antropológicos. A pesar de las dificultades, el trabajo de Dehaene muestra que es posible avanzar -aunque no sabemos hasta dónde- en la primera dimensión. En cuanto a la segunda, la comprensión integral de la conciencia en el contexto global del fenómeno humano no solamente va más allá de la actual ciencia de la conciencia, sino que probablemente rebase el ámbito de la neurociencia misma. 


\section{Referencias}

Aru, J., Bachmann, T., Singer, W., \& Melloni, L. (2012). Distilling the neural correlates of consciousness. Neuroscience and Biobehavioral Reviews, 36, 737-746.

Aru, J., Bachmann, T., Singer, W., \& Melloni, L. (2015). On why the unconscious prerequisites and consequences of consciousness might derail us from unravelling the neural correlates of consciousness. En S. M. Miller (Ed.), The constitution of phenomenal consciousness (pp. 205-225). Amsterdam/Philadelphia: John Benjamins.

Baars, B. (1988). A Cognitive Theory of Consciousness. Cambridge, UK: Cambridge University Press.

Bachmann, T. (2012). How to begin to overcome the ambiguity present in differentiation between contents and levels of consciousness? Forntiers in Psychology, 3, 82. doi: 10.3389/ fpsyg.2012.00082

Block, N. (1978). Troubles with Functionalism. Minnesota Studies in the Philosophy of Science, 9, 261-325.

Block, N. (1995). On a confusion about a function of consciousness. Behavioral and Brain Sciences, 18, 227-287.

Block, N. (2005). Two neural correlates of consciousness. Trends in Cognitive Sciences, 9(2), 46-51.

Block, N. (2011). Perceptual consciousness overflows cognitive access. Trends in Cognitive Sciences, 15(12), 567-575.

Cohen, M. A., \& Dennett, D. (2011). Consciousness cannot be separated from function. Trends in Cognitive Sciences, 15(8), $358-64$.

Crick, F., \& Koch, C. (1990). Toward a neurobiological theory of consciousness. Seminars in Neuroscience, 2, 263-275.

Crick, F., \& Koch, C. (2003). A framework for consciousness. Nature neuroscience, 6, 119-126. doi:10.1038/nn0203-119.

Chalmers, D. (1999). La mente consciente. Barcelona: Gedisa.

Chalmers, D. (2000). What is a neural correlate of consciousness? En T. Metzinger (Ed.), Neural Correlates of Consciousness (pp. 17-39). Cambridge (Mass.): MIT.

Chalmers, D. (2004). How can we construct a science of consciousness? En M. S. Gazzaniga (Ed.), The Cognitive Neurosciences III, third edition (pp. 1111-1120). Cambridge (Mass.): MIT.

Damasio, A. (2010). Y el cerebro creó al hombre. Barcelona: Destino.

De Graaf, T. A., Hsieh, P. J., \& Sack, A. T. (2012). The 'correlates' in neural correlates of consciousness. Neuroscience and Biobehavioral Reviews, 36, 191-197.

De Graaf, T. A., \& Sack, A. T. (2015). On the various neural correlates of consciousness: Are they distinguishable? En S. M. Miller (Ed.), The constitution of phenomenal consciousness (pp. 177-204). Amsterdam/Philadelphia: John Benjamins.

Dehaene, S. (2014). Consciousness and the Brain. Deciphering how the brain codes our thoughts. New York: Viking Penguin.

Dehaene, S., \& Changeux, J. P. (2011). Experimental and Theoretical Approaches to Conscious Processing. Neuron, 70(2), 200-227.

Dehaene, S., Charles, L., King, J. R., \& Marti, S. (2014). Toward a computational theory of conscious processing. Current opinion in neurobiology, 25, 76-84.
Del Cul, A., Dehaene, S., Reyes, P., Bravo E., \& Slachevsky, A. (2009). Causal role of prefrontal cortex in the threshold for access to consciousness. Brain, 132(9), 2531-2540.

Dennett, D. (1996). Commentary on Chalmers: Facing backwards on the problem of consciousness. Journal of Consciousness Studies, 3(1), 4-6.

Dennett, D. (2006). Dulces sueños. Obstáculos filosóficos para una ciencia de la conciencia. Buenos Aires/Madrid: Katz Editores.

Fahrenfort, J. \& Lamme, V. (2012). A true science of consciousness explains phenomenology: Comment on Cohen and Dennett. Trends in cognitive sciences, 16, 138-9.

Haynes, J.D. (2009). Decoding visual consciousness from human brain signals. Trends in Cognitive Sciences, 13(5), 194-202.

Hickok, G. (2014). The Myth of Mirror Neurons: The Real Neuroscience of Communication and Cognition. New York: W. W. Norton and Co.

Hohwy, J. (2009). The neural correlates of consciousness. New experimental approaches needed? Consciousness and Cognition, $18(2), 428-438$

Irvine, L. (2012). New problems with old measures in the science of consciousness. British Journal for the Philosophy of Science, 63, 627-648.

Kay, K. N., Naselaris, T., Prenger, R. J., \& Gallant, J. L. (2008). Identifying natural images from human brain activity. Nature, $452,352-355$.

Kim, J. (2005). Physicalism, or something near enough. Princeton/ Oxford: Princeton University Press.

Kosuch, B. (2014). Prefrontal lesion evidence against higher-order theories of consciousness. Philosophical Studies, 167(3), 721-746.

Levine, J. (1983). Materialism and Qualia: The Explanatory Gap. Pacific Philosophical Quarterly, 64, 354-361.

Lamme, V. A. (2006). Towards a true neural stance on consciousness. Trends in Cognitive Sciences, 10(11), 494-501.

Lamme, V. A. (2010). How neuroscience will change our view on consciousness. Cognitive Neuroscience, 1(3), 204-220.

Meyers, E. M. (2013). The neural decoding toolbox. Frontiers in neuroinformatics, 7(8), 1-12. doi: 10.3389/fninf.2013.00008

Miller, S. M. (2007). On the correlation/constitution distinctionproblem (and other hard problems) in thescientific study of consciousness. Acta Neuropsychiatrica, 9, 159-176. doi: 10.1111/j.1601-5215.2007.00207.x

Miller, S. M. (2015). The correlation/constitution distinction problem: Foundation, limits and explanation in consciousness science. En S. M. Miller (Ed.), The constitution of phenomenal consciousness (pp. 104-154). Amsterdam/Philadelphia: John Benjamins.

Overgaard, M., \& Overgaard, R. (2010). Neural correlates of contents and levels of consciousness. Forntiers in Psychology, 1, 164. doi: $10.3389 /$ fpsyg. 2010.00164

Quian Quiroga, R., Reddy L., Kreiman G., Koch, C., \& Fried, I. (2005). Invariant visual representation by single-neurons in the human brain. Nature, 435, 1102-1107.

Quian Quiroga, R. \& Panzeri, S. (2009). Extracting information from neural populations: Information theory and decoding approaches. Nature Reviews Neuroscience, 10, 173-185. 
Searle, J. R. (2000). Consciousness. Annual Review of Neuroscience, 23, 557-578.

Thirion, B., Duchesnay, E., Hubbard, E., Dubois, J., Poline, J-B., Lebihan, D., \& Dehaene, S. (2006). Inverse retinotopy: Inferring the visual content of images from brain activation patterns. NeuroImage, 33, 1104-1116.
Tononi, G., \& Koch, C. (2008). The Neural Correlates of Consciousness. An Update. Annals New York Academy of Sciences, 1124, 239-261. 


\section{Notas}

1 Siempre que no se indique otra cosa, las referencias bibliográficas se referirán a esta obra, señalándose simplemente el número de página.

2 El espacio global de trabajo fue entendido como una noción cognitivo-funcional para referirse a un sistema cognitivo capaz de difundir e integrar la información (Baars, 1988). En esta arquitectura cognitiva tendríamos, por una parte, procesadores inconscientes de la información especializados en diferentes tareas $y$, por otra, un sistema capaz de hacer que esa información sea accesible para los procesadores especializados o para algunos de ellos. Desde este punto de vista, la conciencia (concebida en términos de conciencia de acceso) coincide con ese sistema de difusión e integración de la información, y la información que accede a este espacio se corresponde con los contenidos conscientes. Esta teoría cognitivo-funcional deja abierta la cuestión de cuál es la implementación neurobiológica concreta del sistema, cuestión a la que Dehaene ofrece una respuesta en este libro.

3 Me refiero a la antropología en un sentido filosófico amplio. La comprensión integral de la conciencia en el contexto global del fenómeno humano (es decir, antropológico) no solamente va más allá de la actual ciencia de la conciencia, sino que probablemente rebasa el ámbito de la neurociencia misma.

4 Aunque estas marcas aparecen expresamente vinculadas a la búsqueda del sustrato cerebral de la conciencia, las signaturas de la conciencia deberían ser entendidas, como se observará más adelante, más como correlatos que como sustratos propiamente dichos. Diferentes actividades o estados cerebrales pueden estar correlacionados con la conciencia, pero eso no significa que se haya identificado (y, aún menos, explicado) el sustrato neuronal, entendido como la actividad o el estado que directa y específicamente constituye (o realiza, produce, causa, etc.) la conciencia. $\mathrm{Si}$ bien es una cuestión que requiere ser conceptualmente elucidada de una manera más profunda, tomo aquí la distinción entre correlato y sustrato como una distinción análoga, en términos generales, a la que se ha propuesto entre correlación y constitución (Miller, 2007, 2015 -lo que no implica asumir necesariamente el detalle de los análisis de Miller-).

5 Donde acceso se refiere a conciencia de acceso y fenomenología a conciencia fenoménica. Como una primera aproximación podemos considerar las definiciones ofrecidas por Cohen y Dennett (2011, p. 358):

-Conciencia de acceso: estados sobre los que se puede informar gracias a funciones cognitivas superiores como la memoria, la atención, y la toma de decisiones.

-Conciencia fenoménica: el aspecto subjetivo de la experiencia del mundo (por ejemplo, la experiencia de ver el color rojo).

-El problema llamado fácil sería el de explicar distintas funciones cognitivas, como el lenguaje y la atención, mientras que el problema difícil sería explicar la conciencia fenoménica.
6 Dehaene, Charles, King y Marti (2014) organizan el mapa conceptual de la conciencia en dos bloques. Distinguen entre contenido de conciencia y estado de conciencia. En relación al el primero señalan cuatro nociones: acceso consciente, procesamiento consciente, informe consciente, y conciencia de sí o autoconciencia. En relación al segundo distinguen entre diversas fluctuaciones en el estado de vigilia (normal, coma, estado vegetativo, anestesia, sueño) y la mera capacidad cerebral de poder tener un flujo de contenidos conscientes. Lo que los autores llaman estado de conciencia es lo que frecuentemente se denomina nivel de conciencia. En la literatura neurocientífica sobre la conciencia es común la distinción entre contenidos y estados o niveles de conciencia (Tononi y Koch 2008 proporcionan un buen ejemplo). Chalmers (2000) propuso distinguir entre correlatos neuronales del estado de fondo de la conciencia (que se corresponden con los niveles) y correlatos neuronales de la conciencia de contenidos específicos.

7 El propio Damasio reconoce que la noción de sí-mismo puede tener distintos significados. En concreto, el sí-mismo como rasgo esencial de la conciencia es en cierto modo prereflexivo y refleja la descripción fenomenológica según la cual "[e]l estado mental consciente se experimenta en la exclusiva perspectiva de cada uno de nuestros organismos en primera persona" (Damasio, 2010, p. 241).

8 Al hablar de la magnitud del debate me refiero, por ejemplo, al alcance de afirmaciones como la de Cohen y Dennett (2011) cuando descalifican explícitamente algunas de las más importantes teorías neurocognitivas de la conciencia al mantener que una teoría de la conciencia que no esté basada en funciones y acceso (o que se base en una división de experiencia y función) no es una teoría científica.

$9 \quad$ El autor solo hace una breve referencia a la vinculación que Lamme (2006, 2010) establece entre procesos recurrentes y conciencia. Según Dehaene, los procesos recurrentes locales serían necesarios, pero no constituirían una condición suficiente para la experiencia consciente, mientras que bucles más amplios (globales) como los propugnados por la teoría del espacio de trabajo neuronal global sí lo serían.

10 Este punto de vista coincide con el de Dennett (1996, 2006).

11 Recordemos que se está hablando de la conciencia de acceso, y que nada ha quedado establecido respecto a la posible epifenomenalidad de los qualia. Uno de los problemas derivados de esta posibilidad sería el de explicar la relación entre los qualia y el informe (verbal o de otro tipo) por parte del sujeto, en la medida en que los qualia carecieran de eficacia causal con respecto a cualquier tipo de informe. Kim (2005) ha defendido un epifenomenalismo parcial, sugiriendo que la función de los qualia es hacer posible la discriminación entre sus cualidades intrínsecas. Mientras que las cualidades intrínsecas, por sí mismas, serían epifenoménicas, las diferencias y semejanzas entre ellas sí tendrían un papel causal, del mismo modo que lo importante en un semáforo no es la cualidad intrínseca de los colores, sino la discriminación entre ellos, ya que el significado que convencionalmente se ha asignado al 
verde podría asignarse al rojo, o un sujeto podría tener invertidos estos colores y seguir actuando correctamente (en este caso, el sujeto tendría una experiencia diferente, pero funcionalmente irrelevante).

12 Esta capacidad de la conciencia para hacer "circular" la información, para hacerla accesible a distintos "módulos" cerebrales, es expresada por el autor mediante la metáfora de la lengua franca. La conciencia proporcionaría, en este sentido, una especie de lengua franca del cerebro que permite compartir e intercambiar información entre distintos sectores especializados, lo que supone una indudable ventaja evolutiva. Una diferencia significativa entre el procesamiento especializado inconsciente y el procesamiento consciente es que mientras que el primero se produce en paralelo, el segundo se realiza en serie, como si se tratara de una máquina de Turing biológica (p. 106). Esta idea parece proponer para la conciencia un modelo computacional clásico (basado en reglas y símbolos) que ha sido ampliamente discutido en filosofía de la mente y en ciencias cognitivas.

13 "Funcional" significa aquí, simplemente, que la conciencia se explica y se justifica por su utilidad cognitiva en la ETNG. Esta utilidad (o función) es la de seleccionar, amplificar y propagar determinada información. "Función" equivale aquí a "tarea" en el sentido en el que, por ejemplo, se afirma que

los cerebros son más que anatomía y fisiología y están diseñados para llevar a cabo ciertas tareas o funciones (...). Cuando los neurocientíficos cognitivos hablan de organización funcional o de sistemas funcionales están refiriéndose a las diferentes tareas que el cerebro lleva a cabo y a cómo los sistemas, redes, o áreas que hacen posibles estas tareas están organizados. (Hickok, 2014, p. $58)$.

14 En la traducción al castellano de la obra se ha vertido "Descifrando el enigma de cómo el cerebro elabora nuestros pensamientos".

15 Lo que parece incluir aquello que usualmente llamamos fenomenología, dimensión cualitativa de la experiencia o, en ocasiones, qualia.

16 La "codificación" de la información implica no solo cierta activación neuronal, sino que las neuronas silentes, las que no se disparar también participan al "informar" de elementos que no están presentes o no son relevantes con respecto a un determinado contenido. En cierto modo, el contraste entre las neuronas activas y las inactivas delinea el contorno de los contenidos conscientes. En palabras del autor, "[e]l acceso consciente talla un pensamiento esculpiendo un patrón de neuronas activas e inactivas en nuestra red de espacio de trabajo global" (p. 181).

17 Patrones de actividad que aparecen si y solo si la persona está teniendo una experiencia consciente (p. 13). A diferencia de las signaturas, los CNC específicos (CE) no tienen por qué ser suficientes y necesarios, ya que una misma experiencia no tiene porqué implicar siempre los mismos correlatos. Chalmers (2000) propone que puede haber más de un CNC (entendido como sistema mínimo suficiente) para un estado mental dado (p. 25) (ver más adelante, nota 20). El punto de vista de Chalmers es compatible con la redundancia neuronal y, en términos filosóficos, con la realización múltiple.

18 Las signaturas se presentan a veces como criterios con respecto al nivel de conciencia (p. 223).

\section{Cf., Searle (2000).}

20 De Graaf et al., distinguen entre correlatos de la experiencia consciente específicos con respecto al contenido y correlatos invariantes, que se dan siempre que se produce cualquier experiencia consciente (como ocurriría con las signaturas). Para estos autores, el método contrastivo no es suficiente, por sí solo, para identificar el sustrato neuronal de la experiencia consciente, ya que no permite distinguir el sustrato de antecedentes y consecuencias de la experiencia. Es decir, dentro de la actividad neuronal que el método del contraste mínimo permite asociar a la experiencia consciente habría que distinguir, en realidad, tres correlatos diferentes -ver también, De Graaf y Sack (2015)-.

21 Haynes (2009) distingue entre dos tipos de condiciones para la conciencia: condiciones de fondo o posibilitadoras, que serían inespecíficas (como la vigilia -wakefulness-), y condiciones específicas que son necesarias para una categoría específica de experiencias conscientes (brillo, color, movimiento). Haynes parece entender aquí los $\mathrm{CNC}$ a un nivel de tipos o clases de experiencias, más que de perceptos individuales específicos.

22 Hay que tener, además, en cuenta que todos estos ejemplos remiten a elementos relativamente simples de la percepción visual, mientras que los contenidos de la conciencia pueden ser más complejos y mucho más abstractos.

23 El conocimiento de estas reglas puede interpretarse de dos modos, uno limitado y otro más profundo. En el modo más profundo ese conocimiento implicaría la resolución del problema difícil de la conciencia, mientras que en el modo limitado bastaría, probablemente, con la identificación de lo que Chalmers (2004) llama principios puente sistemáticos, que explican la covariación de los datos en primera persona y los datos en tercera persona. Según Chalmers, una ciencia de la conciencia podría adoptar una forma no reductiva para la que bastaría con articular la conexión sistemática entre ambos planos (primera y tercera persona) sin reducir uno al otro.

24 Esta noción de correlato nuclear proviene de Chalmers (2000). Hayne (2009) entiende estos correlatos nucleares como aquellos que codifican contenidos específicos de la conciencia y son condición necesaria para los mismos. Desde este punto de vista, el discurso de Dehaene sobre las signaturas de la conciencia parece oscilar entre el sentido del correlato total y el del correlato nuclear (distinción que, a grandes rasgos, se corresponde con la diferencia entre correlatos inespecíficos y correlatos específicos). Aunque la comparación entre Chalmers y Haynes da pie a un análisis más profundo y extenso del que puede hacerse aquí, recordemos que, mientras que el primero considera que los $\mathrm{CNC}$ deben ser mínimamente suficientes pero no 
necesarios y suficientes, Haynes, en lo que podríamos llamar la versión decodificadora de los CNC, entiende que deben ser también necesarios. De este modo, Chalmers admite la posibilidad empírica de que un estado o proceso mental tenga varios correlatos neuronales, mientras que para Haynes cada experiencia o contenido diferente debe corresponderse (formando un mapeo estable) con un estado neuronal diferente. En general, los criterios de Haynes para considerar que un estado (proceso, circuito) neuronal es un CNC parecen ser más estrictos que los de Chalmers.

25 En términos generales la diferencia en el uso de los términos "correlación" y "decodificación" (en el sentido débil, es decir, correlacional), en este contexto, suele implicar que en el segundo caso se pretenden desarrollar métodos de predicción. Pero incluso este tipo de "decodificación" se enfrenta a serias dificultades, sobre todo en el caso de la resonancia magnética funcional, como el hecho de que las correlaciones en que se basa conciernen a estados cerebrales demasiado genéricos, con un nivel de detalle posiblemente insuficiente para capturar toda la riqueza de la vida mental -v., sin embargo, Thirion et al., (2006), Kay et al., (2008), como ejemplos de avances en este terreno-.

26 Por una parte, podemos distinguir entre correlatos-signaturas, como conjunto de meros marcadores o índices, y correlatos como sustrato neuronal. Por otra parte, hay un debate abierto sobre la distinción entre el sustrato o mecanismo de la conciencia de acceso y el sustrato o mecanismo del contenido fenoménico específico (Block 2005 propone distinguir entre correlato neuronal fenoménico y correlato neuronal de acceso).

Dehaene afirma correctamente que la mera correlación no es causación, lo que parece sugerir que entiende las signaturas de la conciencia en términos de causación. Sin embargo, a lo largo del texto aparecen, fundamentalmente, como meros índices de la presencia de acceso consciente. En la distinción entre sustrato o base y mero correlato cabe apreciar dos cuestiones diferentes. Una cuestión se mantiene en el plano de la correlación y trata de la diferencia entre correlatos genuinos (que, en última instancia, apuntan al sustrato), y lo que serían antecedentes y consecuencias (o meras condiciones necesarias posibilitadoras). La segunda cuestión va más allá del plano correlacional y trata del tipo de relación entre fenómenos mentales y fenómenos neurales que hace posible (y, en su caso, explicaría) la correlación. En última instancia, éste sería el plano de la metafísica de la mente: ¿Es la relación subyacente una relación de causación, de superveniencia, de identidad (como apuntan De Graaf et al., 2012), de constitución?

28 Dehaene parece sugerir que la lista de cuatro no es exhaustiva, como se advierte en la aplicación de las signaturas a la detección de conciencia en pacientes con trastornos del estado de conciencia.

29 El papel del cortex prefrontal es una cuestión discutida. El autor concluye, a partir de estudios con estimulación magnética transcraneal, que los lóbulos frontales desempeñan una función causal en los bucles corticales de la conciencia (p. 158). Dehaene y Changeux (2011) reconocen que, a pesar de la evidencia que relaciona la actividad del cortex prefrontal con la percepción consciente, no ha quedado establecido que tenga un papel causal necesario. Kozuch (2014) argumenta en contra de que áreas en esta región sean necesarias para la experiencia visual basándose en diferentes estudios en los que lesiones en el cortex prefrontal no impiden la conciencia en términos de acceso (experiencia visual). Del Cul et al., (2009) señalan que, aunque alteran el umbral perceptivo, lesiones prefrontales no suprimen por completo la ignición distribuida a gran escala que, según el modelo del espacio neuronal global, subyace al acceso consciente.

30 Estas cuestiones ponen de manifiesto el problema de las relaciones entre contenidos, por una parte, y estados o niveles de conciencia, por otra (Searle, 2000; Hohwy, 2009). En particular, en el caso que nos ocupa, el problema de cómo un criterio que básicamente solo distingue entre dos condiciones (según el modelo del contraste mínimo), y basado en última instancia en el informe subjetivo del acceso al contenido, puede ser utilizado para discriminar entre estados o niveles de conciencia (cf., Overgaard \& Overgaard, 2010; Bachmann, 2012). 